\title{
Derepression of Enzyme Synthesis in Response to Arginine Limitation in Neurospora crassa
}

\author{
By HARRY J. FLINT, ${ }^{*}$ SUSAN DIBLE AND HENRIK KACSER \\ Department of Genetics, King's Buildings, University of Edinburgh, Edinburgh EH9 3JN, UK
}

(Received 21 February 1985; revised 26 May 1985)

\begin{abstract}
Ornithine carbamoyltransferase and argininosuccinase, two enzymes involved in arginine synthesis, are regulated by cross-pathway amino acid control in Neurospora and show derepression in response to limitation of any one of a number of amino acids. The effects of varying the severity of arginine limitation upon the synthesis of these enzymes, in mycelial cultures of an arginine auxotrophic strain, are reported here. Derepression occurred at arginine concentrations sufficient to allow normal rates of protein accumulation, leading to increases of not more than fourfold in the absolute rate of enzyme synthesis. On the other hand, differential rates of enzyme synthesis increased progressively up to 20-fold or more under extreme conditions of arginine limitation that also limit net protein synthesis. The major part of the derepression response thus occurred at arginine concentrations that allowed low net rates of protein synthesis. The physiological significance of this is not yet understood. Our evidence suggests that these responses were mediated entirely through the cross-pathway control system, and may not be untypical (allowing for variations in magnitude) of derepression resulting through this mechanism in Neurospora.
\end{abstract}

\section{INTRODUCTION}

The derepression of amino acid synthetic enzymes that occurs under conditions of amino acid limitation has been studied quite extensively in Neurospora, and this has led to a general description of many of the control systems involved (Davis, 1975). There are, however, few, if any, systems for which quantitative information is available relating changes in enzyme synthesis to the severity of amino acid limitation. Such information is clearly of importance in understanding the operation of underlying control mechanisms.

This paper is mainly concerned with the regulation of two of the final three enzymes of arginine synthesis in Neurospora, ornithine carbamoyltransferase (OCTase) and argininosuccinase (ASAase). Both enzymes are known to be regulated by cross-pathway amino acid control (Flint \& Kemp, 1981). This form of control mediates the simultaneous derepression of various enzymes involved in the synthesis of arginine, tryptophan, histidine and lysine in Neurospora in response to a decrease in the intracellular concentration of any one of a number of amino acids (Carsiotis \& Jones, 1974; Carsiotis et al., 1974; Barthelmess, 1982; Flint \& Kemp, 1981). Similar forms of control are also found in other fungi (Bode et al., 1983; Piotrowska, 1980; Schürch et al., 1974). In the case of OCTase it has been shown that derepression elicited by limitation of arginine, tryptophan or histidine can be completely abolished by mutation at the $c p c-1$ locus, which is thought to encode a positively acting regulatory element involved in cross-pathway control (Barthelmess, 1982). This, and the absence of any repressive effect of arginine in wildtype strains (Cybis \& Davis, 1975; Flint \& Kemp, 1981), rules out an additional pathwayspecific form of control, which had been found for OCTase in yeast (Messenguy, 1979), in Neurospora. Evidence presented in this paper suggests that the same applies to ASAase. Thus

Abbreviations: ASAase, argininosuccinase; OCTase, ornithine carbamoyltransferase. 
the derepression of OCTase and ASAase that occurs in response to arginine limitation is attributable to the same mechanism that governs derepression of these enzymes in response to other amino acids and derepression of enzymes in other pathways in response to arginine. We concentrate here on examining derepression under conditions of arginine limitation mainly for reasons of comparability with an earlier study (Barthelmess et al., 1974). However, this system is principally of interest as an example of derepression occurring through the cross-pathway control mechanism.

Two different experimental approaches are used here to study changes in enzyme synthesis resulting from reduced arginine levels. First we compare the effects of gradual arginine depletion in an arginine auxotroph on the absolute rate of OCTase synthesis and on total protein accumulation. Secondly, we examine conditions where the growth of an arginine auxotroph is limited by the continuous supply of low concentrations of arginine. In this case determinations of enzyme activity are used to measure the differential (or relative) rates of enzyme synthesis associated with conditions of arginine limitation of varying severity. These studies provide quantitative information on the relationship of enzyme derepression to the severity of arginine limitation.

\section{METHODS}

Strains. Neurospora crassa strains arg-5a (27947) and his-2a (Y152M14) were obtained from the Fungal Genetics Stock Center, Humboldt State University Foundation, Arcata, Calif., USA. $c p c-1$ arg- $6 a$ is derived from the $c p c-I$ (CD15) and arg-6 (CD25) strains isolated by Davis (1979) and cpc-I his-2a is from cpc-I (CD15) and his-2 (Y175M611). These strains were obtained from I. B. Barthelmess, University of Hannover, FRG. The wild-type strain used was St Lawrence 74a. The arg-5 and arg-6 (CD25) mutations result in the loss of detectable activity for acetylornithine aminotransferase and acetylglutamyl phosphate reductase/acetylglutamate kinase respectively (Davis, 1979), and his-2 affects phosphoribosyl ATP synthetase (Perkins et al., 1982).

Growth conditions. Normal batch cultures were grown at $29^{\circ} \mathrm{C}$ in $200 \mathrm{ml}$ Vogel's liquid medium (Vogel, 1956) containing $2.25 \%(\mathrm{w} / \mathrm{v})$ glucose and appropriate amino acid supplementation in $1-1$ round, baffled flasks. Flasks were shaken at 200 r.p.m. on a gyrorotary shaker. Inocula of $10^{7}$ conidia per flask were prepared from 7- to 14-dold slants. To compensate for the germination lag shown by strains carrying the $c p c-l$ allele, the inoculum was doubled and inoculation advanced by $6 \mathrm{~h}$.

For the experiments involving continuous arginine supply, inoculations were made into $200 \mathrm{ml}$ medium containing a low 'priming' concentration $(0.12 \mathrm{mM})$ of arginine. Flasks were fitted with silicone rubber stoppers carrying a foil capped vent and a glass tube in contact with the medium through which a combined stream of sterile air $\left(0.42 \mathrm{ml} \mathrm{min}^{-1}\right)$ and fresh medium $\left(0.16 \mathrm{ml} \mathrm{min}^{-1}\right)$, containing the appropriate amino acid concentration, was pumped by means of a multichannel proportioning pump. Pumping was continued throughout incubation, while the flasks were shaken at 200 r.p.m. Despite the increase in culture volume that occurs over the period of incubation, this combination of vigorous agitation and forced air ensures adequate aeration throughout, since wild-type strains grow exponentially at a rate independent of the culture volume under these conditions (Flint et al., 1983).

Mycelia were harvested by filtering the complete flask contents through two layers of Whatman no. 1 paper in a Buchner funnel. They were rinsed with a small volume of distilled water, rapidly frozen and then lyophilized and weighed.

Amino acid pools. Arginine was determined using an amino acid autoanalyser on cold $5 \%(\mathrm{w} / \mathrm{v})$ sulphosalicylic acid extracts of powdered lyophilized mycelium as described previously (Flint $e t$ al., 1980).

Enzyme activities. Extracts of powdered, lyophilized mycelium were made by homogenizing with a Teflon/ground glass pestle in $0.05 \mathrm{M}$ - Tris/ $\mathrm{HCl}$ buffer $(\mathrm{pH} 7.5)$ containing $0.1 \mathrm{mM}$-EDTA and $0.5 \mathrm{~mm}$-dithiothreitol, and centrifuging for $20 \mathrm{~min}$ at $1000 \mathrm{~g}$. OCTase (EC 2.1 .3 .3 ) activity was routinely assayed on highly diluted supernatant without dialysis, and other enzymes were assayed after dialysis overnight against $0.01 \mathrm{M}-\mathrm{Tris} / \mathrm{HCl}$ buffer $(\mathrm{pH} 7.5)$ at $4^{\circ} \mathrm{C}$. OCTase activity was assayed by the formation of citrulline, which was estimated colorimetrically. ASAase (EC 4.3.2.1) was assayed by the formation of $\left[{ }^{1+} \mathrm{C}\right]$ argininosuccinate from $\left[{ }^{14} \mathrm{C}\right]-$ arginine (i.e. by the back reaction) as described previously (Flint \& Kemp, 1981). Glutamate acetyltransferase (EC 2.3.1.35) and saccharopine dehydrogenase $\left(\mathrm{NAD}^{+}\right.$, lysine-forming; EC 1.5.1.7) were assayed by the methods of Denes (1970) and Broquist (1971) respectively. Assays were done in duplicate. Total activities (per culture) for OCTase and ASAase were calculated from the activities per unit dry weight extracted and the dry weight harvested. In each case one unit of activity represents the formation of $1 \mu$ mol product $\min ^{-1}$ at $30^{\circ} \mathrm{C}$. Specific activities were obtained after estimation of protein concentration in the extract by the Lowry method.

Total mycelial protein. Powdered, lyophilized mycelium $(20 \mathrm{mg})$ was homogenized in $2 \mathrm{ml}$ cold $5 \%(\mathrm{w} / \mathrm{v}) \mathrm{TCA}$ and centrifuged $(1000 \mathrm{~g}, 10 \mathrm{~min})$ after $30 \mathrm{~min}$ on ice. The precipitate was washed once in $5 \% \mathrm{TCA}$, digested for 


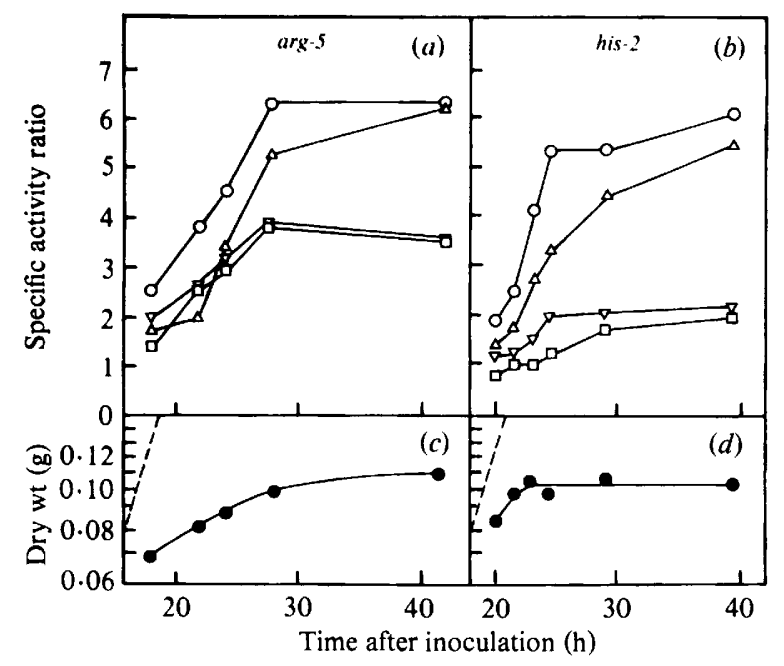

Fig. 1. Growth (dry weight increase) and changes in enzyme specific activity during the later stages of arginine or histidine depletion in amino acid auxotrophs. Conidia (10 $\left.{ }^{7}\right)$ of arg-5 or his-2 (Y 152M14) strains were used to inoculate $200 \mathrm{ml}$ volumes of medium containing, respectively, $0 \cdot 12 \mathrm{~mm}$-arginine or 0.06 mM-histidine. Specific activity ratios (i.e. specific enzyme activities relative to the repressed value determined for a wild-type strain grown on minimal medium) are shown in (a) for $\arg -5$ and in (b) for his-2. OCTase $(O)$, ASAase $(\triangle)$ and glutamate acetyltransferase $(\nabla)$ are all involved in arginine synthesis, and saccharopine dehydrogenase $(\square)$ in lysine synthesis. Absolute specific activity values [nmol (mg protein $)^{-1} \mathrm{~min}^{-1}$ ] for a wild-type strain grown on minimal medium were, respectively, 360 , $8 \cdot 1,29$ and 20. Dry weight increase ( ) for the same cultures is shown on a log scale for $(c)$ arg-5 and $(d)$ his-2. For comparison the exponential growth rates of arg-5 and his-2 during amino acid sufficient growth (on $2 \mathrm{~mm}$-arginine or $0.5 \mathrm{~mm}$-histidine, respectively) are indicated by the dashed lines in (c) and (d) (these were determined from a separate experiment).

$16 \mathrm{~h}$ in $3 \mathrm{ml} 1 \mathrm{M}-\mathrm{NaOH}$, and protein was determined on a 10-fold dilution (in $\mathrm{H}_{2} \mathrm{O}$ ) of the digest by the Lowry method.

\section{RESULTS}

\section{Cross-pathway control of arginine synthetic enzymes}

In the experiments shown in Fig. 1, arg-5 and his-2 auxotrophic strains were allowed to 'run out' of their required amino acid, following supplementation at a low concentration. The progress of specific activity increase, due to derepression, of three arginine synthetic enzymes and of one lysine synthetic enzyme is shown during the later stages of growth. As observed previously (Flint \& Kemp, 1981), OCTase and ASAase showed greater increases in specific activity than the other two enzymes whether arginine or histidine was the limiting amino acid. The derepression of OCTase and ASAase that occurs in response to decreased arginine or histidine was largely abolished in strains carrying the $c p c-1$ (CD15) mutation, impaired in crosspathway amino acid control (Table 1). Although an increase (less than twofold) in both enzymes followed arginine limitation in the $c p c-1$ (CD15) arg-6 strain used here, a previous study using other $c p c-1$ mutant alleles has shown that arginine mediated derepression of OCTase, as well as that of glutamate acetyltransferase and saccharopine dehydrogenase, can be completely abolished by mutation at the $c p c-1$ locus (Barthelmess, 1982). Thus in Fig. 1 the derepression resulting from arginine limitation, as well as from histidine limitation, is probably due entirely to the cross-pathway control system. Table 1 also shows an effect of the $c p c-1$ mutation on basal levels of ASAase and OCTase activity. This has been observed previously for OCTase and for other enzymes subject to cross-pathway control (Barthelmess, 1982). The reason for the slight reduction observed here in ASAase activity upon histidine starvation of the $c p c-1$ his-2 strain (Table 1) is not known. 
Table 1. Effects of a cpc-I mutation on the response of OCTase and ASAase to amino acid limitation

The results are expressed as specific activity ratios, i.e. specific enzyme activities relative to those found for a wild-type strain growing on minimal medium (given in Fig. 1 legend), and are the means of duplicate determinations from a single experiment. Strains carrying arg- 6 were grown on 2 mM-arginine for $16 \mathrm{~h}$ and mycelium was then transferred either to $2 \mathrm{~mm}$-arginine medium for $2 \mathrm{~h}$ (arginine sufficient) or to minimal medium for $8 \mathrm{~h}$ (arginine limited). Strains carrying his- 2 were grown either on $0.5 \mathrm{~mm}$ histidine for $16 \mathrm{~h}$ (histidine sufficient) or on $0.05 \mathrm{~mm}$-histidine for $40 \mathrm{~h}$ (histidine limited). The specific activities under limitation conditions do not necessarily represent maximal values. This, and the fact that the his-2 allele was different from that used in Fig. 1, may account for the lower changes in specific activity observed here for histidine limitation (cf. Fig. 1).

\begin{tabular}{|c|c|c|c|}
\hline \multirow[b]{2}{*}{ Strain } & \multirow[b]{2}{*}{ Growth condition } & \multicolumn{2}{|c|}{ Specific activity ratio } \\
\hline & & OCTase & ASAase \\
\hline $\arg -6$ & $\begin{array}{l}\text { Arginine sufficient } \\
\text { Arginine limited }\end{array}$ & $\begin{array}{l}1 \cdot 26 \\
7.03\end{array}$ & $\begin{array}{l}1 \cdot 14 \\
5 \cdot 73\end{array}$ \\
\hline $\arg -6 c p c-1$ & $\begin{array}{l}\text { Arginine sufficient } \\
\text { Arginine limited }\end{array}$ & $\begin{array}{l}0 \cdot 22 \\
0 \cdot 31\end{array}$ & $\begin{array}{l}0 \cdot 31 \\
0.58\end{array}$ \\
\hline his-2 (Y175M611) & $\begin{array}{l}\text { Histidine sufficient } \\
\text { Histidine limited }\end{array}$ & $\begin{array}{l}0.75 \\
2 \cdot 62\end{array}$ & $\begin{array}{l}0.94 \\
2 \cdot 28\end{array}$ \\
\hline his-2 (Y175M611) cpc-1 & $\begin{array}{l}\text { Histidine sufficient } \\
\text { Histidine limited }\end{array}$ & $\begin{array}{l}0 \cdot 28 \\
0 \cdot 26\end{array}$ & $\begin{array}{l}0 \cdot 72 \\
0 \cdot 39\end{array}$ \\
\hline
\end{tabular}

The period of most rapid increase in specific activity for all four enzymes, during depletion of either amino acid, occurred when growth (dry weight increase) was already severely curtailed (Fig. 1). The progressive restriction of growth would probably limit the final specific activities attained. Changes in specific activity were therefore not expected to provide an accurate measure of the changes in enzyme synthesis that occurred during amino acid depletion.

\section{OCTase synthesis during arginine depletion}

We showed previously that OCTase is stable following shifts from derepressive to repressive conditions, or following inhibition of protein synthesis with cycloheximide, in cultures grown in the presence of glucose and $\mathrm{NH}_{3}$ (Flint \& Kemp, 1981). Furthermore, the immediate curtailment of the increase in OCTase activity that was observed following cycloheximide inhibition in cultures undergoing derepression (Flint \& Kemp, 1981) argues that derepression depends on new protein synthesis rather than activation. Therefore the overall rate of increase in OCTase activity can be used as a measure of the absolute rate of synthesis of this enzyme in cultures undergoing derepression. In Fig. 2 the fully repressed rate of OCTase synthesis is shown for cultures growing exponentially on $2 \mathrm{~mm}$-arginine. A lower concentration of arginine $(0.12 \mathrm{mM})$ resulted in early depletion of the mycelial arginine pool (Fig. $2 \mathrm{~b}$ ) and in cessation of total protein accumulation after $21 \mathrm{~h}$ of growth. (Dry weight increase fell less sharply than protein increase - cf. Figs 1 and 3.) The absolute rate of OCTase synthesis in cultures grown with $0.12 \mathrm{~mm}$-arginine increased 3.5 -fold, by comparison with repressed conditions, at least $3 \mathrm{~h}$ before any change in total protein accumulation was detected (Fig. $2 a$ ). Subsequently enzyme synthesis did not finally cease until at least $5 \mathrm{~h}$ after the apparent cessation of total protein accumulation. This latter observation is probably due to the persistence of a residual level of protein synthesis, resulting from amino acid recycling, together with a high differential (or relative) rate of OCTase synthesis. Protein degradation (at a rate of 4 to $7 \% \mathrm{~h}^{-1}$, from pulse/chase experiments) and protein synthesis (from pulse incorporation of labelled amino acids) continued to be detectable for a considerable period in non-growing, arginine starved cultures (results not shown). 


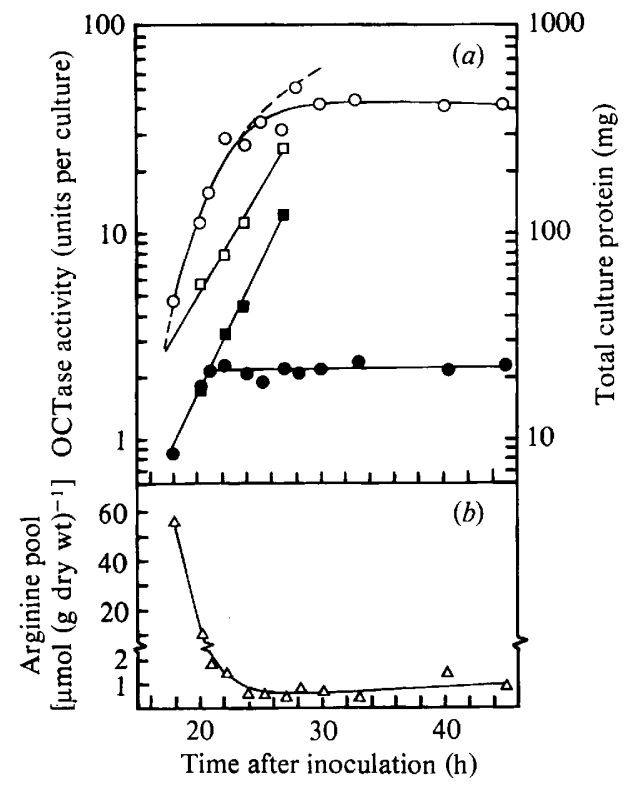

Fig. 2. Total protein and OCTase increase during arginine depletion in an arg-5 strain. (a) Total OCTase activity $(O, \square)$ and total protein $(O, \square)$ were determined in replicate $200 \mathrm{ml}$ cultures inoculated with $10^{7}$ arg-5 conidia, supplemented initially with $2 \mathrm{~mm}$-arginine $(\square, \square)$ or $0 \cdot 12 \mathrm{~mm}$ arginine $(O, O)$. (b) Values for the mycelial arginine pool $(\triangle)$ for the cultures grown with $0.12 \mathrm{~mm}$ arginine. The mean value for the cultures grown with $2 \mathrm{~mm}$-arginine was $85 \mu \mathrm{mol}(\mathrm{gdry} \mathrm{wt})^{-1}$. The dashed line in $(a)$ represents a theoretical curve for the change in OCTase activity in cultures grown with $0.12 \mathrm{~mm}$-arginine, assuming that synthesis occurred at the fully repressed rate up to $17 \cdot 3 \mathrm{~h}$ of growth and at a constant 3.5-fold greater absolute rate [units $\mathrm{h}^{-1}$ (mg protein) ${ }^{-1}$ ] thereafter. It was calculated from $(\mathrm{d} E / \mathrm{d} t)_{t}=p_{t} \cdot 3 \cdot 5 .\left(k . s a_{r}\right)$, where $(\mathrm{d} E / \mathrm{d} t)_{t}$ is the overall rate of enzyme activity increase at time $t$ and $p_{t}$ is the observed total culture protein at this time. The product of the exponential rate constant $(k=0.24)$ and the mean specific activity $\left.\left[s a_{r}=0.269 \text { units (mg protein }\right)^{-1}\right]$ observed for OCTase in cultures grown with $2 \mathrm{mM}$-arginine represents the rate of repressed OCTase synthesis [0.065 units $\mathrm{h}^{-1}$ (mg protein) $)^{-1}$.

\section{Differential rates of enzyme formation during linear amino acid limited growth}

The effects of amino acid limitation on the differential rates of synthesis of OCTase and ASAase were investigated in auxotrophic cultures whose growth was dependent on a continuous low input of amino acid. Since Neurospora mycelium cannot readily be grown in a chemostat, the method used was to supply batch cultures of an arg-5 strain continuously with fresh medium containing defined concentrations of arginine. The cultures received an initial 'priming' level of arginine supplementation $(0.12 \mathrm{mM})$, allowing normal germination and early growth, but subsequently their dry weight increased at a linear rate determined by the concentration supplied (Fig. 3). Linear growth resulted because the constant rate of arginine supply limited the overall rate of protein formation in the culture, regardless of the mycelial mass present. The mycelial protein content per unit dry weight changed little with time once linear growth had become established, although the average value decreased slightly at the lower input levels (Table 2). The arginine pool present in linearly growing mycelium decreased with decreasing supply concentrations (Table 2). The possibility that these conditions also led to significant polyamine limitation, because of the absence of ornithine synthesis in arg-5 strains (Davis et al., 1970), was excluded in preliminary experiments. The linear growth rate of an arg-12 strain (blocked after ornithine), supplied with $0.12 \mathrm{~mm}$-arginine, was approximately $9 \mathrm{mg} \mathrm{h}^{-1}$, and that of an arg-5 strain supplied with $0.12 \mathrm{~mm}$-arginine plus $0.2 \mathrm{~mm}$-putrescine approximately $11 \mathrm{mg} \mathrm{h}^{-1}$ (raw data not shown). This compares with $10 \mathrm{mg} \mathrm{h}^{-1}$ for an arg-5 strain supplied only with $0 \cdot 12 \mathrm{~mm}$-arginine (Table 2). 


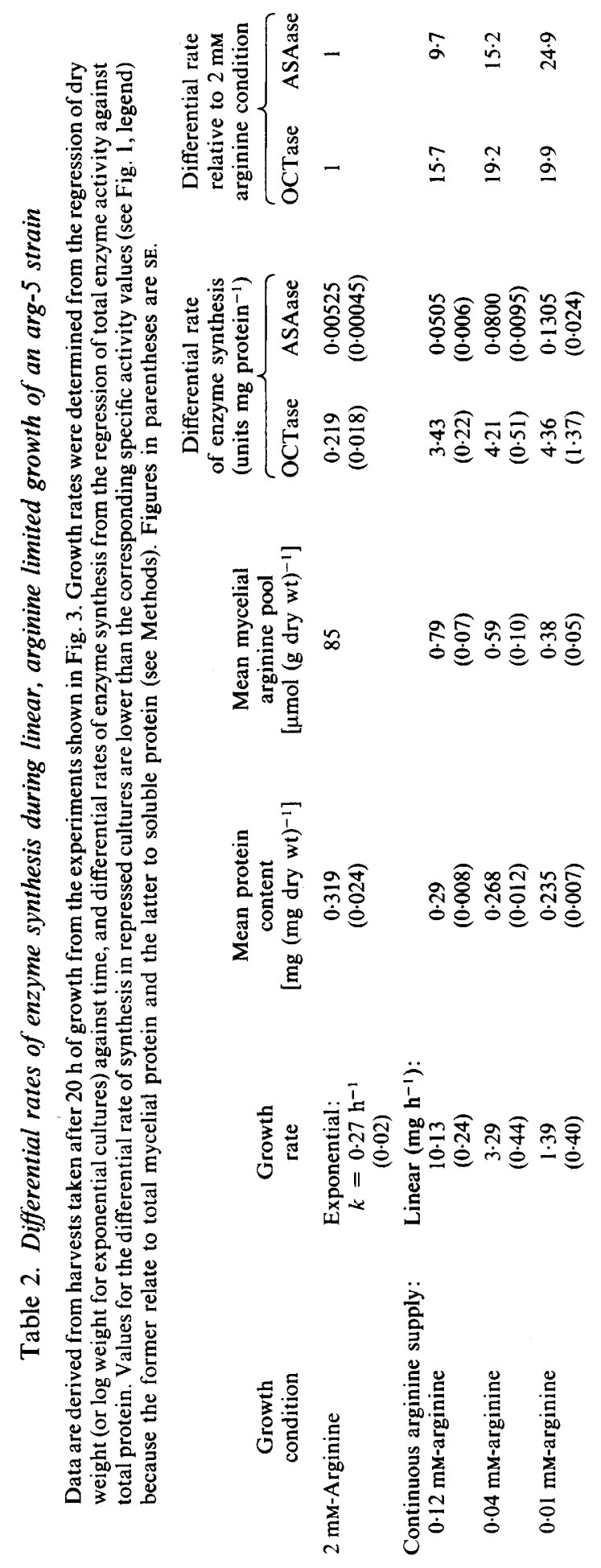




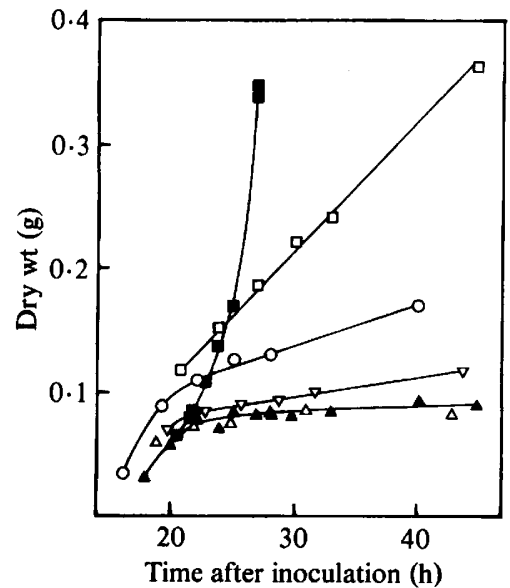

Fig. 3

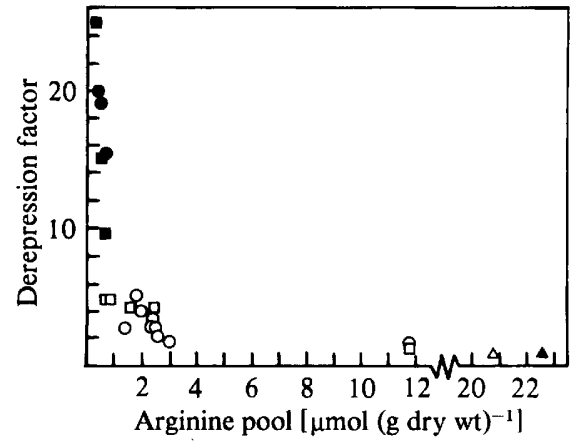

Fig. 4

Fig. 3. Growth of arg-5 batch cultures supplied continuously with arginine. Conidia (10 7 ) of an $\arg -5$ strain were inoculated into $200 \mathrm{ml}$ medium containing $0.12 \mathrm{mM}$-arginine, and supplied continuously from the time of inoculation with fresh medium containing arginine (as described in Methods) at the following concentrations: $0.12 \mathrm{mM}(\square) ; 0.04 \mathrm{mM}(\bigcirc) ; 0.01 \mathrm{mM}(\nabla)$; zero (minimal medium) $(\triangle)$. Cultures that received no supply of fresh medium but were initially supplemented with $0 \cdot 12 \mathrm{~mm}$ arginine $(\boldsymbol{\Delta})$ or $2 \mathrm{~mm}$-arginine $(\square)$ are included for comparison. Each supply concentration represents a separate experiment. At least two cultures supplemented initially with $2 \mathrm{~mm}$-arginine were included in each experiment, receiving inocula identical to those of the supplied cultures. The time scales for different experiments were adjusted to correct for slight variation in the effective inoculum size by visually aligning the parallel log dry wt versus time plots (not shown) of the control cultures grown with 2 mM-arginine.

Fig. 4. Derepression as a function of the total arginine pool. Derepression factors (i.e. differential rates of enzyme synthesis, relative to the repressed value) are shown as a function of the total mycelial arginine pool. Data from the present study (Table 2) for OCTase (O) and ASAase ( $\square$ ) represent conditions of arginine limited growth in an arg-5 auxotroph. Data obtained from arginine bradytrophic strains in an earlier study (Barthelmess et al., 1974) are shown for comparison $(O, \square)$. These were obtained as specific activities relative to repressed values ('specific activity ratios') but are equivalent to differential rates of enzyme synthesis (relative to repressed values) since steady state conditions of exponential growth were used. Values for both enzymes (corresponding to a derepression factor of $1 \cdot 0$ ) for the St Lawrence 74 wild-type grown on minimal medium are also shown ( $\boldsymbol{\Delta}$, present study; $\triangle$, Barthelmess et al., 1974).

During linear growth the total activities (per culture) of OCTase and ASAase increased as approximately linear functions of total culture protein (not shown), allowing estimation of differential rates of enzyme synthesis (given in Table 2). These show a progressive response leading to a 25-fold increase in ASAase synthesis and a 20-fold increase in OCTase synthesis at the lowest input, by comparison with repressed (arginine sufficient) conditions of growth. The difference in the control response of the two enzymes is probably real. ASAase synthesis increased $2 \cdot 5$-fold, while OCTase synthesis increased less than $1 \cdot 5$-fold, between the $0 \cdot 12 \mathrm{~mm}$ and $0.01 \mathrm{mM}$ supply regimes (Table 2). Similar experiments with an arg-6 cpc-1 strain gave values of 0.059 (SE 0.005) and 0.064 (SE 0.005) respectively for the differential rate of OCTase synthesis in $2 \mathrm{~mm}$-arginine grown cultures and $0.12 \mathrm{~mm}$-arginine supplied cultures.

When the derepression factors shown in Table 2 were plotted as a function of the total mycelial arginine pool (which approached stable values during linear growth), there was no inconsistency between these results and those obtained previously by Barthelmess et al. (1974) from arginine bradytrophs during steady state growth (Fig. 4). However, the present results revealed a further sharp increase in derepression at arginine pools slightly below those achieved in the earlier study, i.e. between $2 \%$ and $4 \%$ of the pool of around $20 \mu \mathrm{mol} \mathrm{g}{ }^{-1}$ present in a wildtype strain grown on minimal medium. 


\section{DISCUSSION}

The results of this and previous studies (Barthelmess, 1982; Flint \& Kemp, 1981) indicate that cross-pathway control is the major, if not the only, mechanism responsible for derepression of the arginine synthetic enzymes OCTase and ASAase in Neurospora under conditions of amino acid limitation. Cross-pathway control is known to govern the derepression of enzymes belonging to several different amino acid pathways in response to a reduction in the intracellular concentration of any one of a number of amino acids (Carsiotis et al., 1974; Barthelmess, 1982). This paper provides a quantitative analysis of the response of OCTase and ASAase derepression to a deliberate, progressive reduction of one amino acid pool. Although limitation of one amino acid can lead to increases in many other amino acid pools (Flint \& Kemp, 1981) these are expected to have little influence on the derepression observed here. The levels of amino acids present in wild-type Neurospora grown on minimal medium appear sufficient to exert almost complete repression of OCTase and ASAase (Flint \& Kemp, 1981).

An earlier study (Barthelmess et al., 1974) revealed a progressive increase (up to fivefold) in the specific activity of OCTase and ASAase with decreasing arginine pool size in arginine bradytrophic strains grown exponentially on minimal medium. In the present study, under more severe limitation conditions achieved in an auxotrophic strain, the differential rate of synthesis of these two enzymes increased up to 20 -fold or more.

Previous evidence (Flint \& Kemp, 1981) supports the view that derepression results from a relative increase in enzyme synthesis, due to increased levels of the relevant mRNA species, rather than to activation or decreased enzyme degradation. The differential rates of enzyme synthesis reported here strictly measure net enzyme synthesis relative to net total protein synthesis. As such they could also be influenced by changes in the rate of 'bulk' protein turnover, particularly if this differed from the rates of turnover of the individual enzymes examined. Cultures undergoing arginine starvation in fact showed little increase in the rate of protein degradation by comparison with non-starved, exponential cultures (results not shown). In addition similar differential rates of OCTase synthesis were observed here under conditions of arginine limitation or sufficiency in a $c p c-1$ arg- 6 strain, which is unable to derepress this enzyme, This does not suggest any appreciable effect due to selective degradation. We also found evidence here that net OCTase synthesis could occur in the absence of net protein synthesis in non-growing, arginine starved arg-5 cultures. Under these conditions protein turnover will probably result in some recycling of amino acids, sustaining a basal level of protein synthesis and, presumably, highly derepressed enzyme synthesis.

Derepression factors of the order of 20 -fold due to cross-pathway regulation have not previously been reported in Neurospora. However, much of the available evidence has been derived from examining changes in specific activity after prolonged amino acid starvation of auxotrophs. Our results imply that in an auxotrophic strain differential rates of enzyme synthesis will increase rapidly as amino acid depletion proceeds. Because of the accompanying limitation of protein accumulation, however, the final increase in specific activity underestimates changes in enzyme synthetic rates. This would apply equally to other enzymes showing similar regulatory responses to arginine or other amino acids.

The present results, together with those of Barthelmess et al. (1974), establish that a good correlation exists between enzyme derepression and the total arginine pool under steady state conditions. The relevant signal concentration may not be proportional to the total pool, however, since arginine shows a highly uneven intracellular distribution between cytosol and vacuoles in Neurospora (Subramanian et al., 1973). In addition it has previously been proposed that the level of tRNA charging may provide the signal for derepression occurring via crosspathway control (Spurgeon \& Matchett, 1977). The state of charging of all tRNA species might, for example, be monitored at the level of the ribosome in a manner analogous to stringent control in bacteria (Gallant, 1979). Alternatively a mechanism might exist that simultaneously monitored the charging of many free tRNA species. Our results appear consistent with a signal related to tRNA charging in that derepression was only associated with steady state arginine pools considerably below the level found in a wild-type strain grown on minimal medium. On the other hand, we also showed that OCTase derepression could be triggered initially at an arginine 
concentration that did not detectably limit overall protein accumulation. This could imply that reductions in charging of the relevant tRNAs can signal derepression without appreciably affecting the net rate of protein synthesis.

The observation (Table 2) that differential rates of enzyme synthesis remaired constant during linear, amino acid limited growth, although they responded to variation in supply concentrations, also requires comment. A constant rate of arginine input might be expected to lead to progressively more severe amino acid limitation in such cultures as the mycelial mass and culture volume increase. The apparent approximation to steady state conditions can, however, be explained if the mass of mycelium active in synthesizing protein and in taking up arginine from the medium were constant for a given supply regime, despite the increase in total mass. This situation would arise if these metabolic activities were confined to the extension zones associated with a constant number of hyphal tips growing by linear extension. Both the volume of the extension zone and the number of growing hyphae may decrease with a reduction in the concentration of limiting nutrient supplied (Katz et al., 1972; Trinci, 1978). Steady state conditions did not extend to rates of total RNA and DNA accumulation, which decreased with increasing dry weight as well as with the concentration of limiting nutrient supplied (results not shown).

The physiological role of the responses revealed here has yet to be determined, and it is not known whether the extreme conditions that can be created in auxotrophic strains limited for a single amino acid are encountered by wild-type strains in nature. It is conceivable that the combined effects of simultaneous small reductions in many amino acid pools (or in the charging of many tRNA species) might lead to derepression of the magnitude found here without causing severe growth limitation, but these conditions are not readily achieved experimentally.

We thank Ilse Barthelmess for valuable discussion, and Janice McCormick and Kay Henderson for technical assistance.

This work was supported by a Medical Research Council Grant, no. G8123317CB.

\section{REFERENCES}

BARTHELMESS, I. B. (1982). Mutants affecting amino acid cross-pathway control in Neurospora crassa. Genetical Research 39, 169-185.

Barthelmess, I. B., Curtis, C. F. \& Kacser, H. (1974). Control of the flux to arginine in Neurospora crassa: derepression of the last three enzymes of the arginine pathway. Journal of Molecular Biology 87 , 303-316.

Bode, R., Casper, P. \& Kunze, G. (1983). Induction of the general control of amino acid biosynthesis in Candida spec. EHI5/D using amitrole. Biochemie und Physiologie der Pflanzen 178, 457-468.

Broquist, H. P. (1971). Saccharopine dehydrogenase. Methods in Enzymology 17B, 124-129.

Carsiotis M. \& Jones, R. F. (1974). Cross-pathway regulation: tryptophan mediated control of histidine and arginine biosynthetic enzymes in Neurospora crassa. Journal of Bacteriology 119, 889-892.

Carsiotis, M., Jones, R. F. \& Wesseling, M. (1974). Cross-pathway regulation: histidine mediated control of histidine, tryptophan and arginine biosynthetic enzymes in Neurospora crassa. Journal of Bacteriology: 119, 893-898.

Cybis, J. \& Davis, R. H. (1975). Organisation and control in the arginine biosynthetic pathway of Neurospora. Journal of Bacteriology 123, 196-202.

Davis, R. H. (1975). Compartmentation and regulation of fungal metabolism: genetic approaches. Annual Review of Genetics 9, 39-67.
DAVIS, R. H. (1979). Genetics of arginine biosynthesis in Neurospora crassa. Genetics 93, 557-575.

Davis, R.H., Lawless, M. B. \& PORT, L. A. (1970). Arginaseless Neurospora: genetics, physiology and polyamine synthesis. Journal of Bacteriology 101, 299-305.

Denes, G. (1970). Ornithine acetyltransferase (Chlamydomonas reinhardtii). Methods in Enzymology 17A, 273-277.

Flint, H. J. \& KeMP, B. F. (1981). General control of arginine biosynthetic enzymes in Neurospora crassa. Journal of General Microbiology 124, 129-140.

Flint, H. J., Porteous, D. J. \& Kacser, H. (1980). Control of flux in the arginine pathway of Neurospora crassa: the flux from citrulline to arginine. Biochemical Journal 190, 1-15.

Flint, H. J., Dible, S. \& KaCser, H. (1983). A method for achieving prolonged nutrient limited growth of Neurospora mycelium. Neurospora Newsletter 30, 15.

Gallant, J. A. (1979). Stringent control in E. coli. Annual Review of Genetics 13, 393-415.

Katz, D., Goldstein, D. \& Rosenberger, R. F. (1972). Model for branch initiation in Aspergillus nidulans based on measurements of growth parameters. Journal of Bacteriology 109, 1097-1100.

Messenguy, F. (1979). Concerted repression of the synthesis of the arginine biosynthetic enzymes by amino acids: a comparison between the regulatory mechanisms controlling amino acid biosynthesis in 
bacteria and yeast. Molecular and General Genetics 169, 85-95.

Perkins, D. D., Radford, A., Newmeyer, D. \& BJORKMAN, M. (1982). Chromosomal loci of Neurospora crassa. Microbiological Reviews 46, 426-570.

Piotrowska, M. (1980). Cross-pathway regulation of ornithine carbamoyltransferase synthesis in Aspergillus nidulans. Journal of General Microbiology 116, 335-339.

SCHÜrCH, A., Miozzari, G. \& HÜTter, R. (1974). Regulation of tryptophan biosynthesis in Saccharomyces cerevisiae: mode of action of 5 methyl tryptophan and 5 methyl tryptophan sensitive mutants. Journal of Bacteriology 117, 1131-1140.
Spurgeon, S. L. \& Matchett, W. H. (1977). Inhibition of aminoacyl-transfer ribonucleic acid synthetases and the regulation of amino acid biosynthetic enzymes in Neurospora crassa. Journal of Bacteriology 129, 1303-1312.

Subramanian, K. N., Weiss, R. L. \& Davis, R. H. (1973). Use of external, biosynthetic and organellar arginine by Neurospora. Journal of Bacteriology 115 , 284-290.

TrincI, A. P. J. (1978). Wall and hyphal growth. Science Progress 65, 75-99.

VoGel, H. J. (1956). A convenient growth medium for Neurospora (medium N). Microbial Genetics Bulletin 13, 42-43. 